\title{
Programmable axial apodizing and hyperresolving amplitude filters with a liquid-crystal spatial light modulator
}

\author{
Jeffrey A. Davis \\ Department of Physics, San Diego State University, San Diego, California 92182 \\ Juan Carlos Escalera, Juan Campos, Andres Marquez, and Maria J. Yzuel \\ Departamento de Fisica, Universidad Autonoma de Barcelona, 08193 Bellaterra, Spain \\ Claudio Iemmi \\ Departamento de Fisica, Facultad de Ciencias Exactas y Naturales, Universidad de Buenos Aires, (1428) Buenos Aires, Argentina
}

Received January 26, 1999

\begin{abstract}
Amplitude-transmitting filters for apodizing and hyperresolving applications can be easily implemented by use of a two-dimensional programmable liquid-crystal spatial light modulator operating in a transmissiononly mode. Experimental results are in excellent agreement with theoretical predictions. This approach can permit the analysis of various filter designs and can allow the filters to be changed rapidly to modify the response of an optical system. (C) 1999 Optical Society of America

OCIS codes: $230.6120,110.3000$.
\end{abstract}

We demonstrate that amplitude-transmitting filters for apodizing and hyperresolving applications can easily be implemented with a two-dimensional programmable liquid-crystal spatial light modulator (LCSLM) operating in a transmission-only mode. Experimental results are in excellent agreement with theoretical predictions. This approach can permit the analysis of various filter designs and allow the filters to be changed rapidly to modify the response of an optical system.

Nonuniform amplitude transmission filters can be used to modify the response of optical systems. ${ }^{1-3}$ Different filter designs can produce apodization or hyperresolution either in the transverse plane ${ }^{2,3}$ or along the axis. ${ }^{3,4}$ Here we concentrate on filters of the form $t(r)=a+b r^{2}+c r^{4}+d r^{6}$. These filters were described previously. ${ }^{4-6}$ By changing the values of the coefficients one can generate either transverse or axial apodizing filters.

First we consider an aberration-free system that has a system aperture with a normalized radius $r=1$, as shown by line a in Fig. 1. For this case, the intensity oscillates according to the sinc-squared function shown by curve a in Fig. 2 as the axial position is changed.

The depth of focus (DOF) of the optical system can be increased and the oscillations in intensity in the axial direction can be decreased by use of an axial apodizing filter. Curve b of Fig. 1 shows an example ${ }^{5}$ of such an amplitude-transmitting filter given by $t(r)=$ $6.75 r^{2}-13.5 r^{4}+6.75 r^{6}$. The theoretically calculated intensity along the axis in curve b of Fig. 2 shows that the depth of focus increases and the oscillatory changes in the intensity are smoothed out.

The DOF can also be decreased by use of an axial hyperresolving filter function such as $t(r)=1-4 r^{2}+$ $4 r^{4}$, as shown by curve c of Fig. 1 . This filter not only decreases the DOF but also increases the intensity of the secondary maxima in the axial direction, ${ }^{5}$ as shown by curve c of Fig. 2. Therefore we consider these axial hyperresolving filters as multifocal filters.

In Fig. 2 the three curves are normalized. The relative intensity values in the peak for the axial apodizing filter and the axial hyperresolving filter are 0.316 and 0.11 , respectively. The drawback in using amplitude filters is that the light in the image plane is reduced.

Amplitude pupil filters have been made with photographic emulsions by different techniques. ${ }^{6,7}$ However, these pupil functions can be difficult and expensive to fabricate accurately and cannot be changed quickly. Here we show how to program various transmissive filters by using a programmable liquid-crystal display that operates in an amplitude-only mode.

The experimental system is simple. Light from a $\mathrm{He}-\mathrm{Ne}$ laser with a wavelength of $0.6328 \mu \mathrm{m}$ is expanded, collimated, and sent through an aperture. It is then passed through a twisted nematic LCSLM panel (Sony Model LCX012BL) for the red channel extracted from a Sony Model VPL-V500 video projector. This device has $640 \times 480$ pixels, with a pixel spacing of

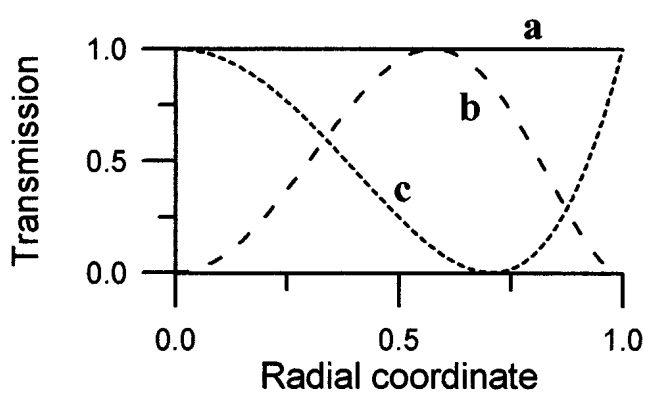

Fig. 1. Amplitude pupil filter functions corresponding to a, uniform transmission; $\mathrm{b}$, an axial apodizing filter, where $t(r)=6.75 r^{2}-13.5 r^{4}+6.75 r^{6} ; \mathrm{c}$, an axial hyperresolving filter, where $t(r)=1-4 r^{2}+4 r^{4}$.

(C) 1999 Optical Society of America 


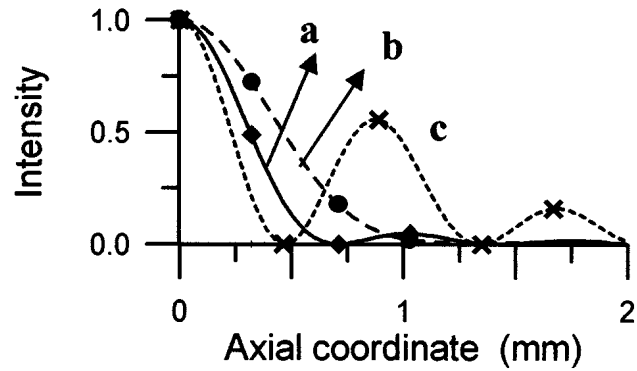

Fig. 2. Theoretically calculated intensity as a function of axial distance for the filters of Fig. 1. Symbols show axial positions where experimental measurements were made: a, uniform transmission; $b$, an axial apodizing filter, where $t(r)=6.75 r^{2}-13.5 r^{4}+6.75 r^{6}$; c, an axial hyperresolving filter, where $t(r)=1-4 r^{2}+4 r^{4}$.

$41 \mu \mathrm{m}$ and a window size of $34 \mu \mathrm{m}$. The aperture that we use has a diameter of approximately 400 pixels.

The light passing through the LCSLM is focused with a $20-\mathrm{cm}$ focal-length lens. The focused spot is magnified with a $40 \times$ microscope objective and imaged onto a CCD camera. The microscope objective also serves as a spatial filter to eliminate higher-order diffracted images caused by the pixel structure of the LCSLM. To examine various defocus planes, we fix the distance between the microscope objective and detector and translate the entire objective-detector system.

The operating parameters of the liquid-crystal display (twist, birefringence, and orientation of director axis) were measured by various techniques. ${ }^{8-10}$ For our device, the twist angle is $-96.3 \mathrm{deg}$, the birefringence is $155.8 \mathrm{deg}$, and the extraordinary birefringence axis is oriented at $47.2 \mathrm{deg}$ relative to the vertical directions. Most researchers who use these devices are interested in either a coupled amplitude and phase modulation or a phase-only modulation. In our case, we want amplitude modulation without any phase modulation. To eliminate phase modulation ${ }^{11}$ we polarize the input light perpendicular to the director axis at the input side (the direction of the ordinary index of refraction), and the output polarizer is perpendicular to the director axis at the output side (which is also the direction of the ordinary index of refraction). In this configuration, there is no phase modulation.

One controls the transmission for the panel by sending a voltage signal to each pixel of the display from a video card in a computer. This video card sends gray-level signals that have values from 0 to 256 . The transmitted intensity is monitored as a function of gray level to calibrate the display. Using these results, we formulate a calibration look-up table of amplitude transmittance $(t)$ versus gray level $(g)$.

As we discussed above, the numerically calculated intensity distributions for the clear aperture and for the two filters are as shown in Fig. 2. These numerical results predict that the axial apodizing filter of Fig. 1, curve b, will increase the DOF and that the axial hyperresolving filter shown in curve c of Fig. 1 will produce multifocal behavior and decrease the DOF. For Fig. 2, the axial coordinate scale was calculated with our experimental parameters.
Figure 3(a) shows the experimental results obtained with a clear aperture. Each three-dimensional (3-D) plot shows the intensity distribution in a different axial plane (these planes coincide with those that are marked in Fig. 2). The sinc-squared behavior of the focused spot intensity is clearly shown. Fortunately, the focused images show no effects of aberrations caused by the LCSLM panel.

Figure 3(b) shows the experimental results for the axial apodizing filter design shown as curve b of Fig. 1. Again, each 3-D plot shows the intensity distribution in a different axial plane. We see that the intensity in the center of the plots decreases more slowly than in the clear aperture [Fig. 3(a)]. Experimental results are in excellent agreement with the theoretical predictions of Fig. 2, curve b.

Figure 4 shows the results for the axial hyperresolving filter of Fig. 1, curve c. In this case the axial intensity diminishes and then increases to form sequential focuses at different axial distances. Figure 4(a) shows the 3-D plots of the intensities, and Fig. 4(b) shows the two-dimensional intensity distributions at several axial positions. Again the positions

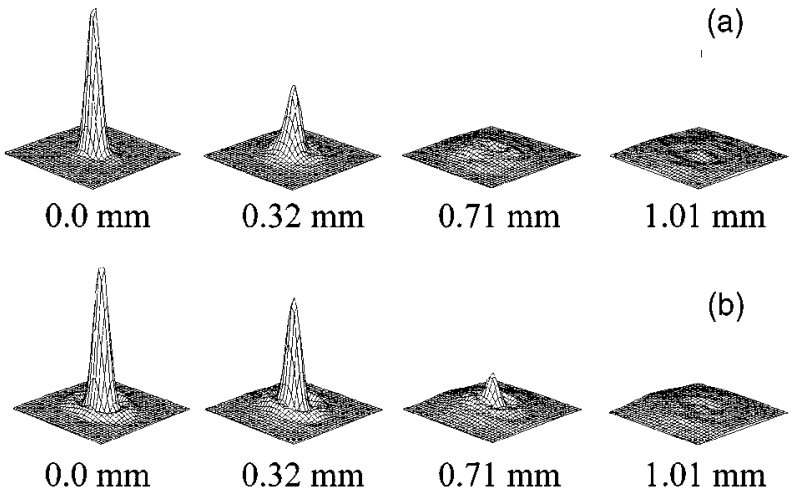

Fig. 3. Experimental measurements of the intensity of the focused spot at the axial positions denoted in Fig. 2: (a) with the uniformly transmitting filter of Fig. 1, line a, the intensity changes according to the expected sincsquared behavior. (b) with the axial apodizing filter of Fig. 1, curve b, the intensity decreases more slowly as a function of axial distance, showing that the DOF has been increased.

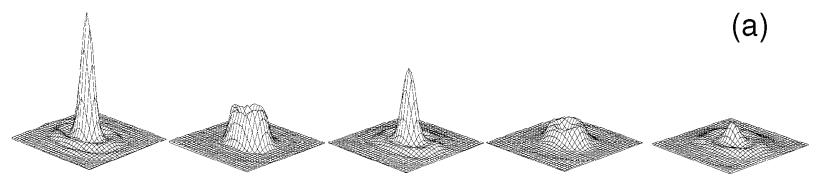

(b)

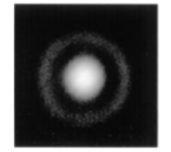

$0.0 \mathrm{~mm}$

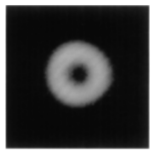

$0.47 \mathrm{~mm}$

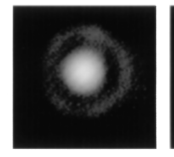

$0.88 \mathrm{~mm}$

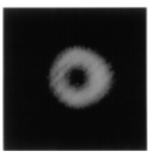

$1.32 \mathrm{~mm}$

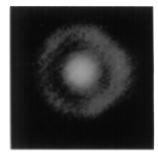

$1.69 \mathrm{~mm}$
Fig. 4. Experimental measurements of the intensity of the focused spot at the axial positions denoted in Fig. 2 with the axial hyperresolving filter of Fig. 1, curve c. The intensity oscillates with axial position, as predicted by theory: (a) 3-D plots at various axial positions; (b) two-dimensional intensity plots at various axial positions. 
of these planes are shown in Fig. 2. The gray levels of Fig. 4(b) have been saturated to show the secondary maxima about the principal maximum. The multifocal behavior of this filter is clearly shown: The intensity drops to zero before increasing to a second and third focus. These results are in excellent agreement with the theory shown in curve c of Fig. 2.

In conclusion, we report the use of an amplitudetransmitting LCSLM to introduce programmable apodizing filters into optical systems. Experimental results for axial apodizing and hyperresolving filters are in excellent agreement with theoretical predictions. The technique is easy to implement and should allow experimental results for various transmissive filters to be obtained easily.

This research was partially financed by the Direccion General de Ensenanza Superior del Ministerio de Educacion y Cultura under project PB96-1134C02-01 and the "Programa de Cooperacion Cientifica con Iberoamerica" (Spain). J. A. Davis gratefully acknowledges the financial support of the Direccion General de Investigacion Cientifica y Tecnica, Ministerio de Education y Cultura (Spain). C. Iemmi gratefully acknowledges the support of the Universidad de Buenos Aires, Consejo Nacional de Investigaciones Cientificas y Tecnicas and Fundacion Antorchas (Argentina). A. Marquez thanks the Comissionat per a
Universitats i Recerca de la Generalitat de Catalunya (Spain) for a grant. J. Campos's e-mail address is ifop8@cc.uab.es.

\section{References}

1. J. P. Mills and B. J. Thompson, J. Opt. Soc. Am. 3, 694 (1986).

2. C. S. Chung and H. H. Hopkins, J. Mod. Opt. 35, 1485 (1988).

3. J. Campos and M. J. Yzuel, J. Mod. Opt. 36, 733 (1989).

4. M. J. Yzuel, J. C. Escalera, and J. Campos, Appl. Opt. 29, 1631 (1990).

5. R. Hild, J. C. Escalera, M. J. Yzuel, and R. Muschall, Pure Appl. Opt. 14, 795 (1995).

6. C. J. R. Sheppard and Z. S. Hegedus, J. Opt. Soc. Am. A 5, 643 (1988).

7. E. W. S. Hee, Opt. Laser Technol. 4, 75 (1975).

8. C. Soutar and K. Lu, Opt. Eng. 33, 2704 (1994).

9. J. A. Davis, D. B. Allison, K. G. D’Nelly, M. L. Wilson, and I. Moreno, "Ambiguities in measuring the physical parameters for liquid crystal spatial light modulators," Opt. Eng. (to be published).

10. J. A. Davis, P. Tsai, K. G. D’Nelly, and I. Moreno, "Simple technique for determining the extraordinary axis direction for twisted nematic liquid crystal spatial light modulators," Opt. Eng. (to be published).

11. K. Lu and B. E. A. Saleh, Opt. Eng. 29, 240 (1990). 